\title{
Staircase of crystal phases of hard-core bosons on the kagome lattice
}

\author{
Daniel Huerga, ${ }^{1}$ Sylvain Capponi, ${ }^{2}$ Jorge Dukelsky, ${ }^{3}$ and Gerardo Ortiz ${ }^{4}$ \\ ${ }^{1}$ Institut für Theoretische Physik III, Universität Stuttgart, Pfaffenwaldring 57, 70550 Stuttgart, Germany \\ ${ }^{2}$ Laboratoire de Physique Théorique, IRSAMC, Université de Toulouse, CNRS, UPS, France \\ ${ }^{3}$ Instituto de Estructura de la Materia, C.S.I.C., Serrano 123, E-28006 Madrid, Spain \\ ${ }^{4}$ Department of Physics, Indiana University, Bloomington, Indiana 47405, USA
}

(Received 26 July 2016; published 12 October 2016)

\begin{abstract}
We study the quantum phase diagram of a system of hard-core bosons on the kagome lattice with nearestneighbor repulsive interactions, for arbitrary densities, by means of the hierarchical mean-field theory and exact diagonalization techniques. This system is isomorphic to the spin $S=1 / 2 \mathrm{XXZ}$ model in presence of an external magnetic field, a paradigmatic example of frustrated quantum magnetism. In the nonfrustrated regime, we find two crystal phases at densities $1 / 3$ and $2 / 3$ that melt into a superfluid phase when increasing the hopping amplitude, in semiquantitative agreement with quantum Monte Carlo computations. In the frustrated regime and away from half-filling, we find a series of plateaux with densities commensurate with powers of $1 / 3$. The broader density plateaux (at densities $1 / 3$ and $2 / 3$ ) are remnants of the classical degeneracy in the Ising limit. For densities near half-filling, this staircase of crystal phases melts into a superfluid, which displays finite chiral currents when computed with clusters having an odd number of sites. Both the staircase of crystal phases and the superfluid phase prevail in the noninteracting limit, suggesting that the lowest dispersionless single-particle band may be at the root of this phenomenon.
\end{abstract}

DOI: 10.1103/PhysRevB.94.165124

\section{INTRODUCTION}

Frustrated systems are distinguished by the subtle interplay between crystal geometry and interaction among its microscopic components [1]. This interplay may lead to stabilization of a plethora of competing thermodynamic phases, some of which are characterized by exotic orders. Particularly interesting is the response of Mott-or any other strongly interacting-insulating materials to applied magnetic fields at low temperatures, since very often the system transitions between commensurate and incommensurate phases, each signaled by the presence of a magnetization plateau as the field changes [2].

These so-called (classical or quantum) frustrated magnets are effectively described by spin degrees of freedom distributed in a graph or lattice, such as kagome, interacting through Heisenberg or Ising-type interaction terms [3]. Eventually, plaquette or ring-exchange interactions may become relevant [4]. In Ising or easy axis models of the magnet, the system may display a complete devil staircase [5], i.e., a series of magnetization plateaux where wide plateaux alternate with quasi-infinite series of smaller ones, with characteristic correlation lengths large but not infinite. On the other hand, in Heisenberg models quantum fluctuations can induce melting of those small plateaux states into a long-range ordered phase, leading to an incomplete staircase. To assess this melting phenomenon is hard, both from the theoretical and experimental standpoints. From the theoretical side, large system sizes are needed, while high NMR resolution and very clean samples are experimentally desired [5].

Recent numerical studies of the kagome Heisenberg antiferromagnetic model (KHAF) found a series of plateaux at magnetizations commensurate with nine units of the saturation magnetization [6-8]. In particular, the crystal phase state just below saturation can be exactly represented by a localized resonant magnon over a background of fully polarized spins [9]. At zero magnetic field, the KHAF is a paradigmatic example of a frustrated quantum magnet and a prominent candidate for hosting a translational invariant paramagnetic ground state, so-called quantum spin liquid. The KHAF is conjectured to be realized in recently synthesized herbertsmithite, a layered compound that shows the absence of magnetic order for very low temperatures [10], although the true nature of its excitations remains elusive due to the presence of impurities [11,12]. Latest experiments on samples with 5\%-10\% concentration of impurities show a finite spin excitation gap [13]. Understanding the response to external magnetic fields will help to unveil the physics of its excitations.

Motivated by experimental efforts to synthesize new magnetic materials, in this paper we investigate the quantum phase diagram, and nature of the low-lying excitations, of a general class of frustrated magnets that includes spin anisotropy. We consider a kagome lattice with $\mathcal{N}$ sites (vertices) where on each vertex $j$ lies a quantum spin, $S=1 / 2$, described by the operator $S_{j}^{v}(v=x, y, z)$, interacting with its nearest-neighbor spin $i$, defining the link $\langle i j\rangle$. Its model Hamiltonian is given by

$$
H=\sum_{\langle i j\rangle}\left[J\left(S_{i}^{x} S_{j}^{x}+S_{i}^{y} S_{j}^{y}\right)+\Delta S_{i}^{z} S_{j}^{z}\right]-h \sum_{j} S_{j}^{z},
$$

where $h$ represents the external magnetic field and the exchange interaction along the $z$ quantization axis is always taken to be antiferromagnetic $(\Delta \geqslant 0)$. Parameter values include ferromagnetic (FM), $J<0$, and antiferromagnet (AFM), $J>$ 0 regimes. For $\Delta=J$ and $h=0$, Eq. (1) reduces to the $\mathrm{SU}(2)$ symmetric Heisenberg model. For any other point in the phase diagram, this "XXZ model" possesses global U(1) symmetry corresponding to rotations of spin operators in the $x y$ plane. Since current ultracold atom technology may allow for a clean quantum simulation of this model [14], it is appropriate and convenient to analyze its isomorphically equivalent hard-core 


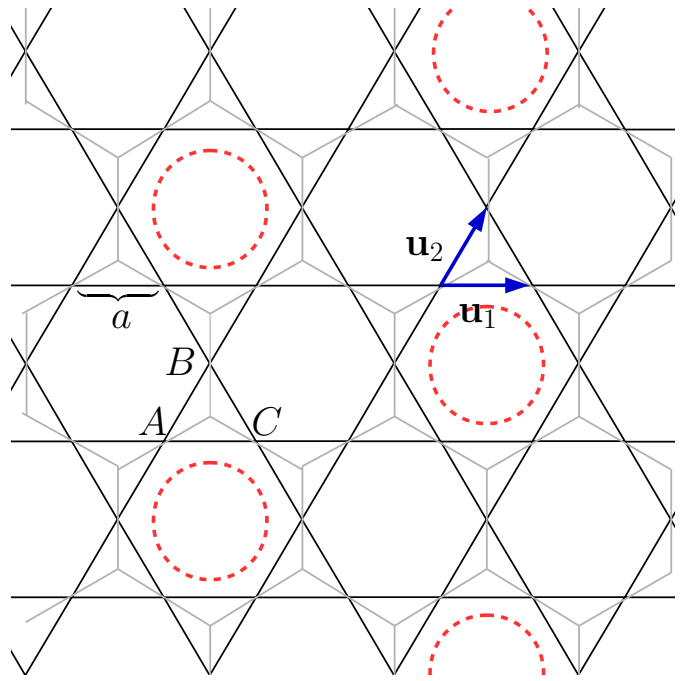

FIG. 1. Schematics of a kagome lattice (black lines) and the underlying honeycomb lattice defined by the centers of the triangular plaquettes (gray). Blue arrows mark the two basis vectors of the kagome lattice, $\mathbf{u}_{1}$ and $\mathbf{u}_{2}$. Capital letters label the three-site basis $A, B, C$. Red dashed circles indicate the pattern of the localized resonant hole valence bond crystals (VBCs), as explained in the text.

boson model with nearest-neighbor repulsive interactions, as will be shown below.

Before proceeding with our main findings let us briefly summarize current knowledge about the XXZ model (1), which can be re-written in terms of the total spin of a corner-sharing triangle (see Fig. 1). Up to an irrelevant additive constant, the Hamiltonian takes the form

$$
H=\frac{1}{2} \sum_{p}\left[J \mathbf{S}_{p}^{2}+(\Delta-J)\left(S_{p}^{z}\right)^{2}-h S_{p}^{z}\right]
$$

where $\mathbf{S}_{p}=\sum_{j \in p} \mathbf{S}_{j}$ and $S_{p}^{z}=\sum_{j \in p} S_{j}^{z}$ are the total spin and its $z$ component of triangle $p$, respectively. In the Ising limit, $J=0$, and $h=0$, the exact ground-state manifold is defined by all spin configurations with $S_{p}^{z}= \pm 1 / 2$. When applying a magnetic field $(h>0)$, this degeneracy is reduced as the exact ground-state manifold is defined by those configurations with $S_{p}^{z}=+1 / 2$. The number of configurations coincides with all hard-core dimer coverings of the honeycomb lattice [15]. A small but finite value of the $\mathrm{XY}$ anisotropy further lifts the degeneracy favoring valence bond crystal (VBC) states characterized by a fully packed pattern of localized threemagnon resonant states over a background of fully polarized spins, in a pattern represented by dashed red lines in Fig. 1. This pattern was found to characterize the $\mathrm{VBC}_{\rho}$ phases at $\rho=1 / 3$ and $2 / 3$ in the FM regime by quantum Monte Carlo (QMC) computations on the isomorphically equivalent hardcore boson model [16].

At precisely the SU(2) Heisenberg point (i.e., the KHAF), (2) becomes a sum of local projectors over the subspace with $S_{p}=3 / 2$,

$$
H=J \sum_{p} P_{p}^{3 / 2}
$$

up to an irrelevant constant, implying that any state containing a singlet in a triangle satisfies locally the energy constraint. The ground-state manifold is thus defined by those many-body states minimizing the number of frustrated triangles. In this regime, as QMC computations are affected by the signproblem, different analytical and numerical approaches have been used to unveil the nature of the ground state. Projection onto the short-range resonant valence-bond subspace $[17,18]$ has been used to study the proliferation of the low-lying singlet states found by exact diagonalization (ED) [19]. Series expansions around the dimer limit [20] found a VBC with a 36site unit cell. Density matrix renormalization group (DMRG) computations find a translational invariant ground state with an excitation gap [21] and $\mathbb{Z}_{2}$ topological order [22,23], while other methods have proposed different translational invariant gapped [24,25] or gapless ground states [26].

In presence of a magnetic field, the KHAF has been studied by means of ED [6], DMRG [7] and, more recently, by infinite projector entangled pair states (iPEPS) [8]. These works find a series of magnetization plateaux at values of the magnetization $M$ commensurate with nine (in units of the saturated magnetization), $M=1 / 9,1 / 3,5 / 9,7 / 9$, with $1 / 3$ representing the broader plateau. While the exact nature of the $M=1 / 9$ plateau state is still debated-existing proposals argue for either a topological state [7] or a more conventional VBC state [8] - the wave functions for the other three plateaux states $(M=1 / 3,5 / 9,7 / 9)$ can be approximately described by hexagon magnon states distributed over a background of fully polarized spins in the fully stacked pattern described above [6,7]. In particular, the one-resonant magnon state approximating the $M=7 / 9$ plateau is the exact ground state of model (1) previous to saturation [9].

As anticipated, we may rewrite the XXZ model (1) in terms of hard-core bosons by applying the Matsubara-Matsuda isomorphism to the SU(2) spin operators [27]. Explicitly, the ladder operators of the $S=1 / 2$ representation, $S_{j}^{ \pm}=$ $S_{j}^{x} \pm \mathrm{i} S_{j}^{y}$, are mapped to creation and annihilation hard-core boson operators, $S_{j}^{+}=a_{j}^{\dagger}$ and $S_{j}^{-}=a_{j}$, and the Cartan to the number operator $n_{j}=a_{j}^{\dagger} a_{j}, S_{j}^{z}=n_{j}-1 / 2$, leading to

$$
H=t \sum_{\langle i j\rangle}\left(a_{i}^{\dagger} a_{j}+\text { H.c. }\right)+V \sum_{\langle i j\rangle} n_{i} n_{j}-\mu \sum_{j} n_{j}+\mathrm{C},
$$

where

$$
t=J / 2, \quad V=\Delta, \quad \mu=h+2 \Delta, \quad \mathrm{C}=\mathcal{N}(\Delta+h) / 2 .
$$

The chemical potential $\mu$ controls the total hard-core bosons density, $\rho$, the nearest-neighbor density-density interaction is repulsive $(V \geqslant 0)$ and frustrated, and the hopping amplitude $t$ is tuned from the nonfrustrated $(t<0, \mathrm{FM})$ to the frustrated $\left(t>0\right.$, AFM) regimes. ${ }^{1}$

The total spin of the corner-sharing triangles partitions the quantum phase diagram in terms of $\left(S_{p}, S_{p}^{z}\right)$ pairs which

\footnotetext{
${ }^{1}$ This terminology is often used since the model is amenable to sign-free QMC simulations but we should emphasize that the densitydensity interaction is always frustrated.
} 


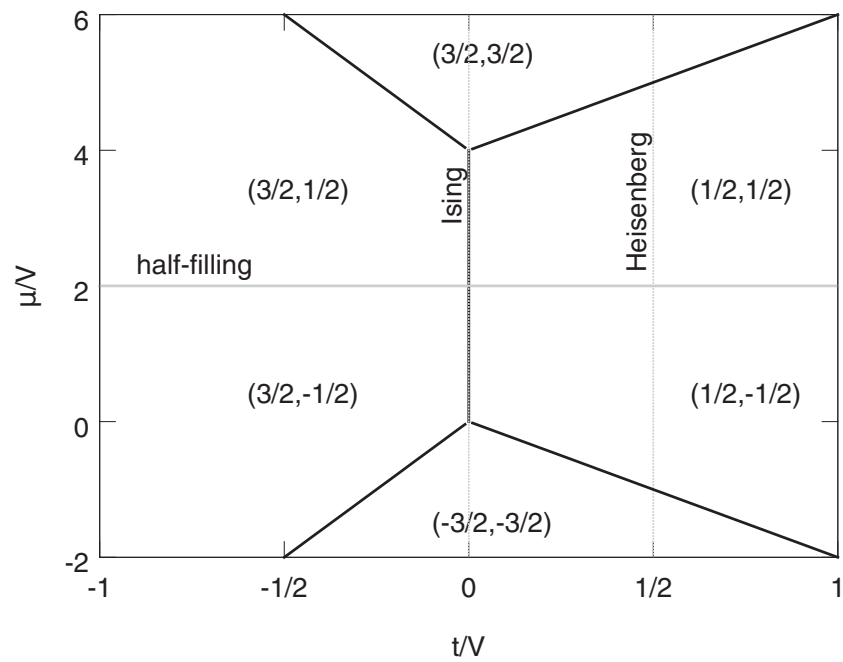

FIG. 2. Parameter regimes of the hard-core boson model on the kagome lattice (4). Regions are classified by the total and third component of the spin of a triangle $\left(S_{p}, S_{p}^{z}\right)$ that locally minimize the equivalent XXZ Hamiltonian written in the honeycomb lattice defined by the centers of the triangles comprising the kagome (2). Solid black lines correspond to parameters where the Hamiltonian has an exact solution. Dotted gray lines correspond to $h=0$ (half-filling), Ising, and Heisenberg limits, as explained in the text.

minimize locally the Hamiltonian, as shown in Fig. 2. At vanishing external magnetic field $(\mu=2 \Delta)$ the spin version (1) corresponds to half-filling in its bosonic counterpart, where a particle-hole symmetry holds. In particular, the AFM XY limit translates into a tight-binding model of hard-core bosons characterized by a dispersionless lowest-energy band. The Ising and Heisenberg limits (arbitrary $h$ ) are thus placed at $t / V=0$ and $t=V / 2$, respectively. The global $\mathrm{U}(1)$ symmetry of the spin rotations in the $x y$ plane is translated into the conservation of the total number of bosons. The density of hard-core bosons is related to the relative magnetization by

$$
\rho=\frac{M+1}{2},
$$

once taken into account the three-site basis of the kagome lattice. Thus, the magnetization plateaux encountered in previous works have a direct translation to the density of hard-core bosons,

$$
\begin{aligned}
& M=\{1 / 9, \quad 1 / 3, \quad 5 / 9, \quad 7 / 9\} \\
& \uparrow \uparrow \uparrow \uparrow \\
& \rho=\{5 / 9, \quad 2 / 3, \quad 7 / 9, \quad 8 / 9\} .
\end{aligned}
$$

In this work, we aim at providing a unified description of the complete quantum phase diagram, including the Ising, Heisenberg, and the FM and AFM XY limits of the hard-core boson model (4), or equivalently the XXZ model (1), by means of the hierarchical mean-field theory (HMFT) [28-32] and ED. The HMFT is a versatile algebraic framework based on the use of clusters of the original degrees of freedom as the basic building blocks containing the short-range correlations, which account for the main features of the phases present in

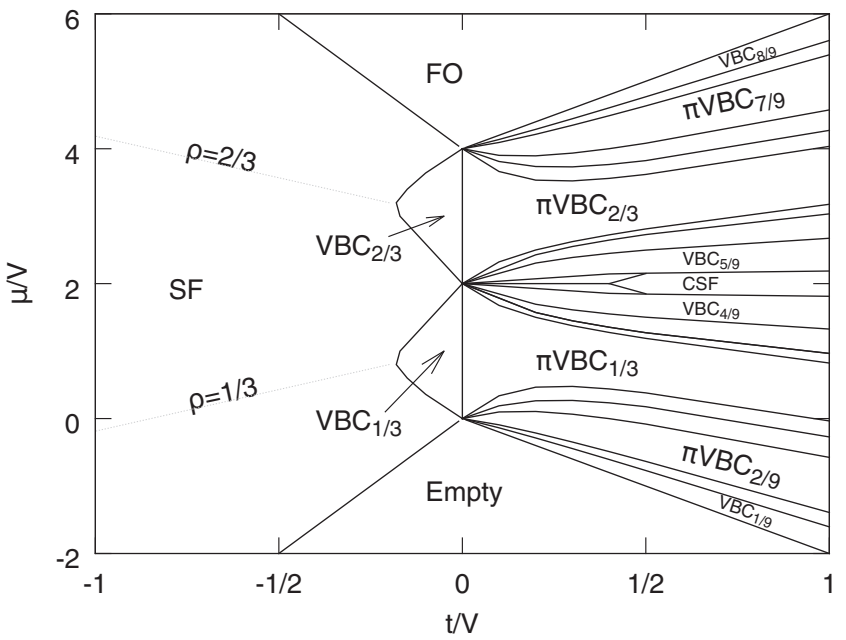

FIG. 3. Quantum phase diagram of the hard-core boson model (4) as obtained within HMFT using 27-site clusters (referred in the text as 27-HMFT). Phases are labeled in capital letters as: fully occupied (FO), superfluid (SF), chiral superfluid (CSF) and valence bond crystal of density $\rho\left(\mathrm{VBC}_{\rho}\right)$. The prefix $\pi$ is used to distinguish those VBCs with real positive amplitudes in their wave functions, from those containing some negative ones. Nonlabeled phases correspond to crystal phases with densities commensurable with the 27 -site, but not with the nine-site cluster. Dotted gray lines correspond to constant density lines within the SF phase.

the system under study. Following this idea, cluster states are represented as the action of a composite boson $(\mathrm{CB})$ over a new vacuum. As the relation between the original spin, or bosonic operators, and the new ones can be cast in a canonical form, we can rewrite the Hamiltonian of interest in terms of CBs and approach it by standard techniques, with the advantage that short-range quantum correlations are taken into account exactly from the onset. To further support our findings, we will also perform ED calculations on finite clusters with periodic boundary conditions (PBC).

Our main findings are summarized in Fig. 3. In the nonfrustrated regime (FM), we obtain two $\mathrm{VBC}_{\rho}$ lobes of densities $\rho=1 / 3$ and $2 / 3$ that melt into a superfluid (SF) in semiquantitative agreement with previous QMC computations [16]. We have reproduced these QMC data by simulating the model using the stochastic series expansion (SSE) algorithm [33,34] of the ALPS library [35]. Moreover, the superfluid order parameter (i.e. stiffness) was computed following the steps of Ref. [36], where a precise method for computing this quantity was put forward for non-Bravais lattices, a case often overlooked in the literature.

In the frustrated regime - or, equivalently, AFM — and away from half-filling, we obtain the series of $\mathrm{VBC}_{\rho}$ main plateaux of densities $\rho$ commensurate with $1 / 9$ as previously described. The prefix $\pi$ is used to distinguish the wave functions from the nonfrustrated region. Around half-filling $(\mu / V \sim 2)$ and hopping amplitudes $t>0.4 \mathrm{~V}$, the staircase of crystal phases melts into a superfluid characterized by the breakdown of the global U(1) symmetry and onset of Bose-Einstein condensation (BEC). For clusters with an odd number of sites, this superfluid possesses nonvanishing chiral currents (CSF) and is doubly degenerate; each state displaying a current 
of opposite chirality. For clusters with an even number of sites, e.g., a 18-site cluster, the chirality vanishes. This is consistent with previous ED studies [19] performed at the SU(2) Heisenberg point, where the first $S=1$ excitation was found to have nonzero Chern number only in clusters with an odd number of sites. We find this nontrivial "odd-even effect" along the whole $t / V>0$ axis. This superfluid region considerably diminishes its size upon increasing the cluster size, indicating that it might perhaps only survive at $\mu=2 \mathrm{~V}$ in the thermodynamic limit. Note that chiral phases have been found to be competing states at half-filling, meaning that they can be stabilized by longer-range interactions [37] or explicit chiral interactions [38], and also in the absence of SU(2) symmetry [39].

For hopping amplitudes $0<t<0.4 \mathrm{~V}$, the half-filling line defines a first order transition between VBCs with densities $\rho=13 / 27$ and $14 / 27$. In-between plateaux, we obtain a series of narrower plateaux commensurate with $1 / 27$ which diminish in width, discarding the possibility of the onset of a standard superfluid order characterized by BEC at momentum $\mathbf{k}=\left(k_{x}, k_{y}\right)$ consistent with the 27 -site cluster. Nonetheless, we cannot asses their stability in the thermodynamic limit, as they were obtained with a single coarse-graining. This is in partial disagreement with the findings of Ref. [8] at the Heisenberg line, where a nine-site unit cell was used and some regions between main plateaux where claimed to support $\mathrm{U}(1)$ breakdown, while others were not fully characterized.

All the quantum phases we found essentially prevail in the XY limit, consistent with similar conclusions obtained at zero magnetic field (half-filling) in Refs. [40,41]. In particular, the $\mathrm{VBC}_{8 / 9}$ is exact at precisely the phase boundary in the whole AFM regime, in agreement with Ref. [9]. Similarly, we found the $\rho=7 / 9$ plateau $\left(\pi \mathrm{VBC}_{7 / 9}\right)$ to be described by a pattern of fully stacked resonant localized-magnons from the Ising to the AFM XY regime, finding no evidence of additional degeneracies that would support either a VBC with further degeneracy — as claimed in Ref. [8] — or a topologically ordered state-as claimed in Ref. [42]. From our ED and HMFT results, the $\rho=2 / 3$ plateau $\left(\pi \mathrm{VBC}_{2 / 3}\right)$ smoothly transitions from a fully stacked localized resonant-magnon $\mathrm{VBC}$ (similar to the $\mathrm{VBC}_{8 / 9}$ and $\pi \mathrm{VBC}_{7 / 9}$ ) to a more complex VBC pattern in the AFM XY limit. Based on our analysis of the low-lying ED spectrum of finite clusters, we cannot exclude the possibility that, in this limit, the $\rho=2 / 3$ plateau becomes either a translational invariant gapped state [42], or a gapless BEC. Similarly, we find the $\rho=5 / 9$ plateau $\left(\pi \mathrm{VBC}_{5 / 9}\right)$ to be a complex VBC in the whole AFM regime. Again, we cannot exclude the possibility it becomes a translational invariant threefold degenerate gapped state in the XY limit [7].

The outline of the paper is as follows. In Sec. II, we briefly describe the HMFT approach at zero temperature and some details about the ED computations, together with the order parameters and observables used to characterize the different quantum phases. In Sec. III, we present the complete quantum phase diagram and provide a detailed analysis of the quantum correlations in the various crystal and superfluid phases encountered in both the frustrated and nonfrustrated regimes. Finally, Sec. IV concludes with a summary and outlook.

\section{THEORETICAL METHODS}

In this section, we provide a brief description of the two methods used in this work: HMFT in the Gutzwiller approximation, and ED of finite clusters. From a technical standpoint, each method emphasizes a different aspect of the infinite-size (thermodynamic) limit solution and thus their combined use provide complementary information.

The HMFT in the Gutzwiller approximation involves the iterative diagonalization of a finite cluster of size $N$ with open boundary conditions (OBC) and a set of selfconsistently defined mean fields acting on its boundaries. The CB Gutzwiller wave functions are exact ground states previous to saturation. In the rest of the phase diagram, while shortrange correlations within the clusters are computed exactly, the mean-fields carry information about the thermodynamic limit allowing for potential breakdown of symmetries and the concurrent stabilization of long-range ordered phases. These CB Gutzwiller wave functions permit the description of quantum phases characterized by the onset of long-range superfluid order, signaled by the occurrence of BEC at various momenta, as well as crystal or superfluid phases characterized by chiral currents [32,43].

On the contrary, ED consists of a single diagonalization performed on a finite cluster with PBC. Since lattice symmetries and conservation of the total number of bosons (or, equivalently, magnetization) can be exploited to reduce the dimension of the Hilbert space, one can simulate cluster sizes beyond those used in HMFT. In the ED case, assessing the stability or absence of long-range order is determined by a finite-size scaling analysis.

\section{A. Hierarchical mean-field theory}

The HMFT is an approach based on the use of a cluster as the basic degree of freedom. In practice, the original lattice is tiled with clusters such that every site belongs to a unique cluster, preserving the original symmetries of the problem as much as possible. The quantum states of each cluster $|\alpha\rangle_{\mathbf{R}}$ are represented by the action of a bosonic (CB) operator over a vacuum $|0\rangle$,

$$
b_{\mathbf{R}, \alpha}^{\dagger}|0\rangle \equiv|\alpha\rangle_{\mathbf{R}},
$$

where $\alpha$ labels the quantum state of the cluster in a generic basis, and $\mathbf{R}$ its position in the cluster superlattice. In this way, quantum correlations with a range smaller than the size of the cluster are taken into account exactly, and thus local information about different competing orders is unbiased. The mapping relating the original bosons to the new $\mathrm{CBs}$ is canonical whenever the latter satisfy the Schwinger constraint $[30,44]$,

$$
\sum_{\alpha} b_{\mathbf{R}, \alpha}^{\dagger} b_{\mathbf{R}, \alpha}=1, \quad \forall \mathbf{R},
$$

which is equivalent to demand that any many-body state of the original problem can be written, in terms of CBs, as a linear combination of product states with one CB per cluster. As the mapping is canonical, the original Hamiltonian can thus be rewritten in terms of these new CBs and solved by standard many-body techniques. The method is variational whenever 


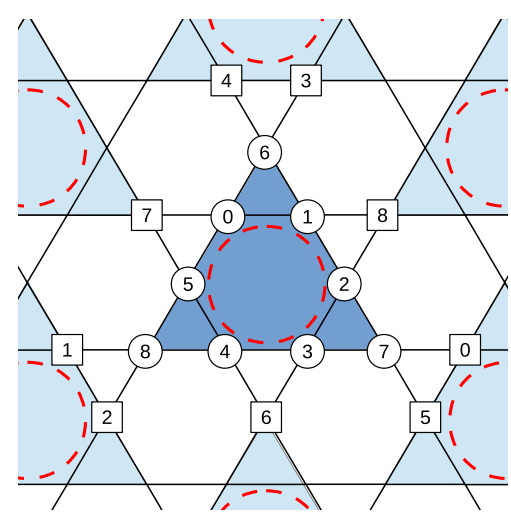

(a)

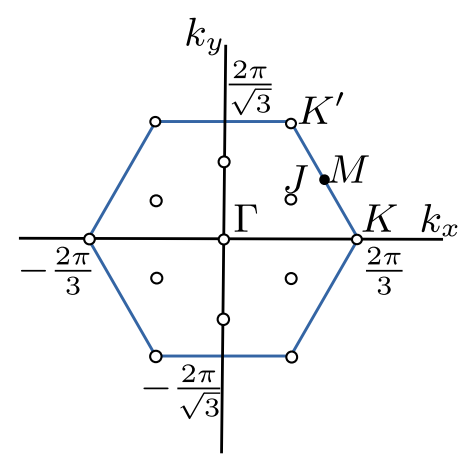

(b)

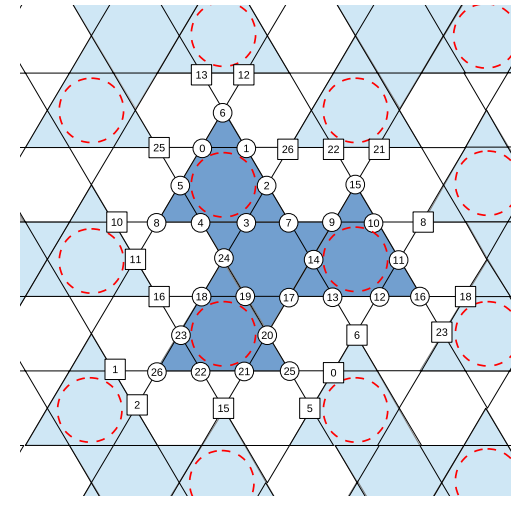

(c)

FIG. 4. (a) and (c) Schematic pictures showing the (a) nine-site and (c) 27-site cluster tilings used in the HMFT approach of this work. Both tilings contain the fully packed resonant-hole hexagon pattern of the exact $\mathrm{VBC}_{8 / 9}$ state, represented by dashed red lines. Numbers label the lattice sites within the cluster. Circles represent intra-cluster sites, while squares represent those sites where the auxiliary mean fields are evaluated. (b) First Brillouin zone of the kagome lattice. Empty circles mark the momenta (k) consistent with the nine-site and 27-site tilings. In particular, the 9-HMFT contains $\Gamma, K$, and $K^{\prime}$ points, while the 27-HMFT contains in addition the $J$ point and its rotations. The $M$ point, identified with a solid circle, is contained within the 36-site cluster used for ED.

the constraint is fulfilled exactly. However, proposing a generic ansatz satisfying the Schwinger constraint exactly is a nontrivial task common to all slave-particle approaches.

In this work, we use a homogeneous Gutzwiller wave function of CBs (CB Gutzwiller), which satisfies the Schwinger constraint exactly,

$$
|\Psi\rangle=\prod_{\mathbf{R}}|\Phi\rangle_{\mathbf{R}}, \quad|\Phi\rangle_{\mathbf{R}}=\sum_{\mathbf{n}} U_{\mathbf{n}} b_{\mathbf{R}, \mathbf{n}}^{\dagger}|0\rangle,
$$

where $\mathbf{n}$ refers to the quantum state in the occupation basis. The amplitudes $U_{\mathbf{n}}$ are determined by making stationary the expectation value of the Hamiltonian (4). The resulting set of nonlinear equations can be cast in a Hartree eigensystem form which is solved iteratively until self-consistency is reached. This homogeneous Gutzwiller approach is equivalent to perform ED on a finite $N$-site cluster, with $\mathrm{OBC}$ and a set of self-consistent auxiliary fields acting on the boundaries of the cluster, where the Hartree or mean-field cluster Hamiltonian has the form

$$
H_{\mathrm{mf}}=H^{\square}+H^{\times}(\{\psi, \eta\}),
$$

with superscripts $\square$ and $\times$ referring to intra- and intercluster terms, respectively. The inter-cluster terms depend upon a set of self-consistently defined mean-fields $\{\psi, \eta\}$ that rely on the particular form of the Hamiltonian and the tiling performed. In the present case,

$$
\begin{aligned}
H^{\square}= & t \sum_{\langle i j\rangle \in \square}\left(a_{i}^{\dagger} a_{j}+\text { H.c. }\right) \\
& +V \sum_{\langle i j\rangle \in \square} n_{i} n_{j}-\mu \sum_{j \in \square} n_{j},
\end{aligned}
$$

with sums running over sites within the same cluster, and

$$
H^{\times}=t \sum_{\langle i, j\rangle}\left(a_{i}^{\dagger} \psi_{j}+\text { H.c. }\right)+V \sum_{\langle i, j\rangle} n_{i} \eta_{j},
$$

where $\langle i j\rangle$ represent the intercluster links connecting a cluster site $i$ and a site $j$ belonging to the neighboring cluster. The set of auxiliary fields are defined as

$$
\psi_{j}^{*}=\left\langle\Phi\left|a_{j}^{\dagger}\right| \Phi\right\rangle, \quad \eta_{j}=\left\langle\Phi\left|n_{j}\right| \Phi\right\rangle,
$$

where we have dropped the superlattice index $\mathbf{R}$, as in the homogeneous CB Gutzwiller wave function all clusters are equivalent. These auxiliary fields are evaluated on the boundaries of the embedding clusters. In Figs. 4(a) and 4(c), we show schematic pictures of the two main tilings used in this work, i.e., nine-site and 27-site clusters, both containing exactly the fully packed localised resonant-hole pattern of the exact $\mathrm{VBC}_{8 / 9}$ previously described.

The CB Gutzwiller wave function (9) allows systematic computation of order parameters and observables. In particular, as it satisfies the Schwinger constraint exactly, it provides a variational upper bound to the energy and phase boundaries are established by monitoring any nonanalytic behavior in its derivatives. In addition, we computed the total density, the condensate density of hard-core bosons, signaling the breakdown of global U(1) symmetry and onset of BEC, the bond currents signaling the breakdown of time-reversal symmetry, and the expectation value of the local hoppings on the links.

The total density is simply defined as the average value of the density in the cluster,

$$
\rho=\frac{1}{N} \sum_{j \in \square}\left\langle n_{j}\right\rangle .
$$

From the macroscopic eigenvalues of the density matrix one can determine the condensate density [45]. Due to translational symmetry, the density matrix is diagonal in momentum space,

$$
\rho_{\alpha, \beta}^{\mathrm{c}}(\mathbf{k})=\frac{1}{\mathcal{N}_{S}}\left\langle a_{\mathbf{k}, \alpha}^{\dagger} a_{\mathbf{k}, \beta}\right\rangle,
$$

where $\mathcal{N}=3 \mathcal{N}_{s}$ is the total number of sites of the lattice and $\mathbf{k}$ refers to a vector within the first Brillouin zone. The kagome lattice is a triangular Bravais lattice with a three-site basis. Each point in the lattice is determined by the triangular 
lattice vector $\mathbf{r}$ and the two basis vectors $\mathbf{u}_{1}, \mathbf{u}_{2}$ (see Fig. 1). When computed with the CB Gutzwiller ansatz (9), the density matrix becomes

$$
\begin{aligned}
\rho_{\alpha, \beta}^{\mathrm{c}}(\mathbf{k})= & \frac{9}{\mathcal{N}^{2}} \sum_{\mathbf{r} \in \square}\left\langle a_{\mathbf{r}+\mathbf{u}_{\alpha}}^{\dagger}\right\rangle e^{-i \mathbf{k}\left(\mathbf{r}+\mathbf{u}_{\alpha}\right)} \\
& \times \sum_{\mathbf{r}^{\prime} \in \square}\left\langle a_{\mathbf{r}^{\prime}+\mathbf{u}_{\beta}}\right\rangle e^{i \mathbf{k}\left(\mathbf{r}^{\prime}+\mathbf{u}_{\beta}\right)},
\end{aligned}
$$

to order $1 / \mathcal{N}_{s}$, where the sums run over the sites of a given species inside the cluster. For each $\mathbf{k}$ point, we further diagonalize in the species subspace, and we can define the total condensate density at $\mathbf{k}$ as the sum

$$
\rho^{\mathrm{c}}(\mathbf{k})=\sum_{\tilde{\alpha}} \rho_{\tilde{\alpha} \tilde{\alpha}}^{\mathrm{c}}(\mathbf{k})
$$

where $\tilde{\alpha}$ refers to the diagonal basis in the species space. At each point in the Brillouin zone, two of the three eigenvalues of (16) are null and the third is positive, in both 9-HMFT and 27-HMFT,

The bond-currents can be defined through the Heisenberg equation for the local number operator, $\mathrm{i} \partial n_{j} / \partial t=\left[n_{j}, H\right]$, in units of $\hbar$. By requiring the local density to be a conserved quantity, $\left[n_{j}, H\right]=0$ one can define local bond currents satisfying $\sum_{\langle i j\rangle} \mathcal{J}_{i j}=0$ for a given site $i$,

$$
\mathcal{J}_{i j}=\frac{\mathrm{i}}{2}\left(a_{i}^{\dagger} a_{j}-\text { H.c. }\right) \text {. }
$$

This quantity is the $z$ component of the vector spin chirality [46], $\kappa_{i j}^{z}=\left(\mathbf{S}_{i} \times \mathbf{S}_{j}\right)^{z}$, when written in terms of hard-core bosons. When taking the expectation value of the bond-current operator, one can distinguish between two cases, i.e., when the bond is contained within the cluster,

$$
\left\langle\mathcal{J}_{i j}\right\rangle^{\square}=\frac{\mathrm{i}}{2}\left(\left\langle a_{i}^{\dagger} a_{j}\right\rangle-\text { c.c. }\right),
$$

and when the bond is connecting two different clusters after the tiling,

$$
\left\langle\mathcal{J}_{i j}\right\rangle^{\times}=\frac{\mathrm{i}}{2}\left(\psi_{i}^{*} \psi_{j}-\text { c.c. }\right),
$$

where $i \in \square$ and $j \in \square^{\prime} \neq \square$. The occurrence of nonzero bond currents is associated with complex-valued self-consistent auxiliary fields.

Similarly, the expectation value of the hopping operator,

$$
B_{i j}=\frac{1}{2}\left(a_{i}^{\dagger} a_{j}+\text { H.c. }\right),
$$

depends on whether the bond lies within the cluster or connecting the two clusters.

\section{B. Exact diagonalization}

We use a standard Lanczos algorithm to compute the density as a function of the chemical potential-equivalent to the magnetization curve in the spin language-for various cluster sizes. As discussed in more detail in Ref. [6], in order to accomodate the exact $\mathrm{VBC}_{8 / 9}$ state, we restrict ourselves to clusters containing the $K$ points of the Brillouin zone, i.e., clusters with 27, 36, and 45 sites, as shown in Fig. 5.

We characterize the quantum phases by computing various correlation functions related to the observables previously

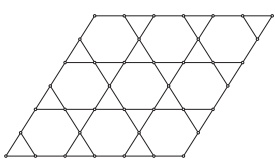

(a)

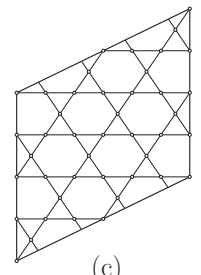

(c) (b)

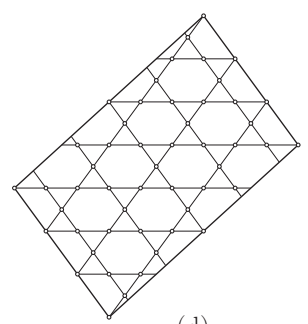

(d)

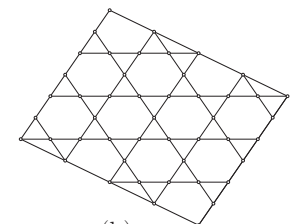

FIG. 5. Clusters used for the ED computations in this work: (a) 27, (b) 36c, (c) 36, and (d) 45 .

defined. In particular, we compute the connected kinetic correlations,

$$
\left\langle B_{i j} B_{k l}\right\rangle_{c}=\left\langle B_{i j} B_{k l}\right\rangle-\left\langle B_{i j}\right\rangle\left\langle B_{k l}\right\rangle,
$$

where $i j$ and $k l$ denote pairs of nearest-neighbor sites comprising the bonds. Analogously, we compute current correlations to detect time-reversal symmetry breaking and the onset of chirality,

$$
\left\langle\mathcal{J}_{i j} \mathcal{J}_{k l}\right\rangle_{c}=\left\langle\mathcal{J}_{i j} \mathcal{J}_{k l}\right\rangle-\left\langle\mathcal{J}_{i j}\right\rangle\left\langle\mathcal{J}_{k l}\right\rangle
$$

We also compute the fidelity susceptibility $\chi_{F}$ to establish whether a quantum phase transition, not captured by the HMFT approach, takes place at fixed density when varying the density-density interaction strength $V[47,48]$. This quantity detects phase transitions without any a priori knowledge of the order parameter, when varying a driving parameter, by measuring the change in the ground-state wave function $\left|\psi_{0}\right\rangle$. In our case,

$$
\left|\left\langle\psi_{0}(V) \mid \psi_{0}(V+\delta V)\right\rangle\right| \simeq 1-\frac{1}{2} \chi_{F}(\delta V)^{2} .
$$

\section{QUANTUM PHASE DIAGRAM}

The quantum phase diagram is obtained by computing the ground state energy and its derivatives, together with the observables and order parameters defined in the previous section. We assess the stability of the phases obtained with the nine-site cluster CB Gutzwiller ansatz (9-HMFT) by performing a second coarse-graining with a 27 -site cluster (27HMFT). In the first case, all $2^{9}=512$ cluster configurations are considered in the Hartree optimization (10). In the second case, we introduce a cutoff in the number of states considered due to the extremely large Hilbert space dimension. We adjust this cutoff depending on the value of $\mu$ controlling the total density of the system. In particular, in-between the main plateaux we have used cluster configurations satisfying the condition $\rho_{\text {low }} \leqslant \rho \leqslant \rho_{\text {ab }}$, where $\rho_{\text {low }}$ and $\rho_{\text {ab }}$ refer to the densities of the immediate below (low) and above (ab) plateaux. Near half-filling, we keep those states with $12 \leqslant$ $N_{\square} \leqslant 15, N_{\square}=\rho N$, summing up to 74884320 states and 


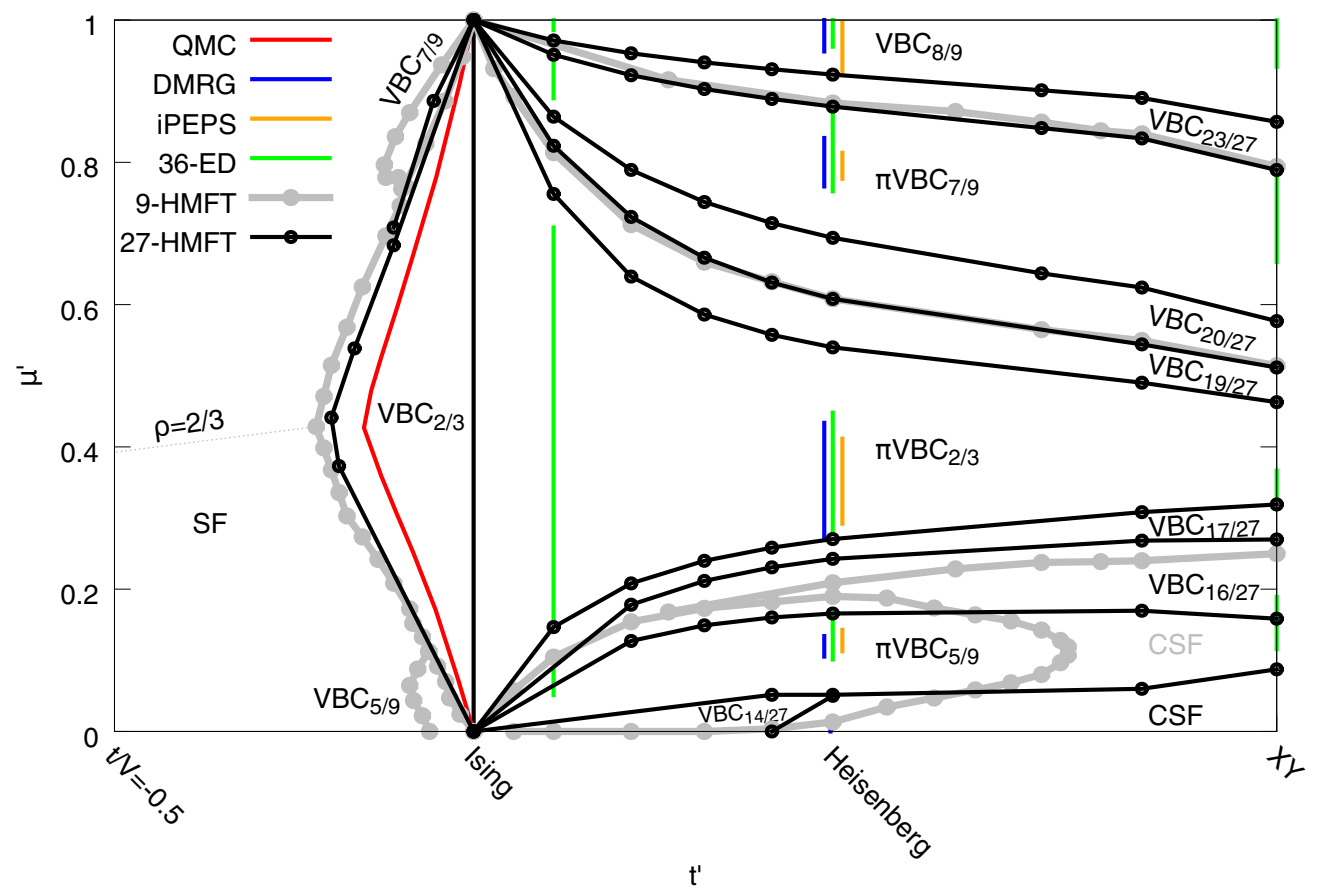

FIG. 6. Quantum phase diagram of model (4) computed with a nine-site (gray) and 27-site (black) CB Gutzwiller ansatz and ED. Also shown are QMC results from Ref. [16] (red), and DMRG and iPEPS plateaux widths at the Heisenberg line $(t / V=+0.5)$ from Ref. [7] (blue) and Ref. [8] (orange), respectively. The normalized chemical potential $\left(\mu^{\prime}\right)$ and hopping amplitude $\left(t^{\prime}\right)$ axis are defined to fit all physical limits. Phases are labeled as superfluid (SF), chiral superfluid (CSF) and valence bond crystal of density $\rho$ (VBC $\rho$ ). The prefix $\pi$ distinguishes frustrated from nonfrustrated regimes. The constant $\rho=2 / 3$ line within the SF phase (dashed) is computed with 9-HMFT. Due to particle-hole symmetry, the diagram is symmetric around the half-filling line $\mu^{\prime}=0$. The trivial fully occupied and empty phases, correspond to $\mu^{\prime}>1$ and $\mu^{\prime}<-1$, respectively.

allowing for a density range $4 / 9 \leqslant \rho \leqslant 5 / 9$. A wider density range is available with increasing chemical potential, e.g., above the $\pi \mathrm{VBC}_{2 / 3}$ plateau, where we have used $18 \leqslant N_{\square} \leqslant$ 27 . We have verified that it does not affect the final results, within error bars, by choosing different cutoffs for a given region in the phase diagram.

Figure 6 displays the phase diagram obtained with 9-HMFT (gray lines) and 27-HMFT (black lines) together with the plateaux obtained with ED on 36-site clusters (36-ED), in green (see Sec. III B 2) for different hopping strengths, as well as QMC results [16], DMRG [7], and iPEPS [8] in the Heisenberg limit. We have normalized the hopping amplitude and the chemical potential in order to fit all parameter regimes within a single plot,

$$
t^{\prime}=\frac{t}{\sqrt{t^{2}+V^{2}}}, \quad \mu^{\prime}= \begin{cases}\frac{\mu-2 V}{2(V-2 t)}, & t / V<0, \\ \frac{\mu-2 V}{2(V+t)}, & t / V>0 .\end{cases}
$$

Note that the phase diagram is symmetric around the halffilling line $\left(\mu^{\prime}=0\right)$ due to particle-hole symmetry, thus we only plot the $\rho \geqslant 1 / 2$ region.

\section{A. Nonfrustrated regime $(t<0)$}

In the nonfrustrated region, we find a superfluid (SF), the trivial fully occupied (FO), and a $\mathrm{VBC}_{2 / 3}$ phase. We also observe two small lobes of density $\rho=5 / 9,7 / 9$ around $\mu^{\prime}=0$ and $\mu^{\prime}=1$, respectively, that shrink considerably upon increasing the cluster size, and presumably vanish in the thermodynamic limit. This is in agreement with previous analytical [49,50] and numerical [16] studies where a stable SF phase for any $t<0$ at half-filling was found.

The $\mathrm{VBC}_{2 / 3}$ wave function obtained within the 9-HMFT approach is dominated by configurations containing three localized resonant holes,

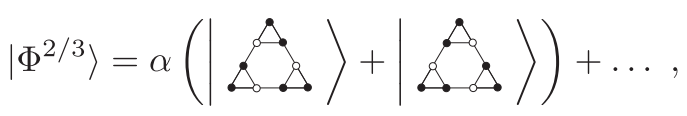

where we refer to occupied (empty) sites as black (white) dots. For small hoppings, $-0.1 V<t<0, \alpha^{2} \sim 0.49$.

The SF phase is characterized by a finite condensate density at the $\Gamma(\mathbf{k}=0)$ point of the Brillouin zone. The phase transition from the SF to the $\mathrm{VBC}_{2 / 3}$, at constant density $\rho=2 / 3$, is found to be second order (see Fig. 7), in qualitative agreement with previous QMC studies [16,51], where it was argued to be weakly first order after a refined scaling and histogram analysis [16].

The phase boundary between the SF and FO phase can be exactly determined by equating the energy of the local Hamiltonian (2) for $\left(S_{p}, S_{p}^{z}\right)=(3 / 2,1 / 2)$ and $(3 / 2,3 / 2)$, leading to

$$
\mu_{\text {full }}(t<0)=4(V-t)
$$

It could be equally computed noticing that a unique delocalized hole over a background of fully occupied sites is indeed an exact eigenstate of the Hamiltonian (4). 


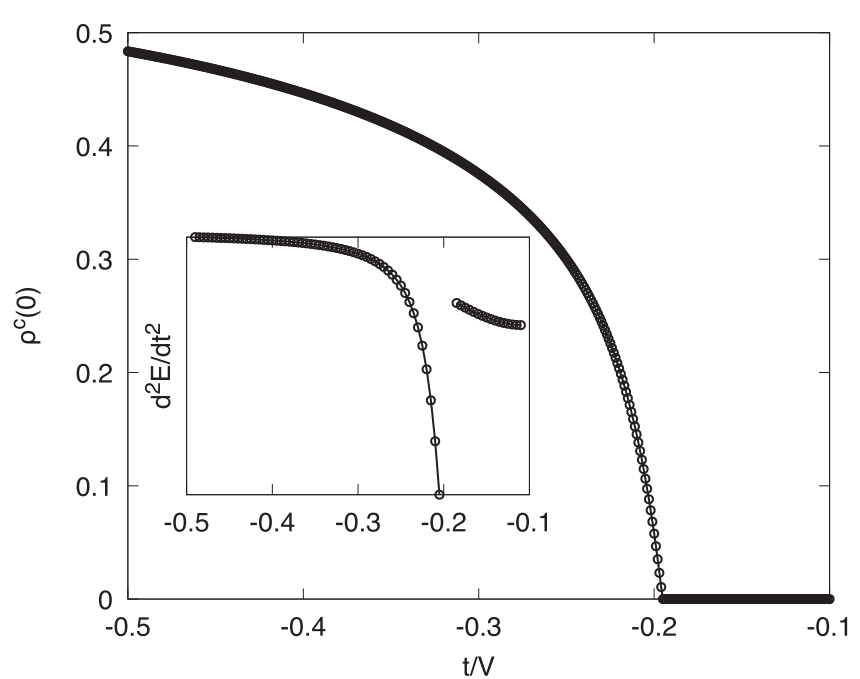

FIG. 7. Condensate density computed with a nine-site $\mathrm{CB}$ Gutzwiller along the $\rho=2 / 3$ density line, and across the SF-VBC $2 / 3$ transition in the nonfrustrated regime of the phase diagram (Fig. 6). (Inset) Second-order derivative of the ground-state energy along the same line.

\section{B. Frustrated regime $(t>0)$}

Similarly, in the frustrated regime, the boundary to the FO state is determined by finding the degeneracy point of the local energies for the $(1 / 2,1 / 2)$ and $(3 / 2,3 / 2)$ local Hamiltonian (2),

$$
\mu_{\text {full }}(t>0)=2(2 V+t),
$$

or by equating the exact energies of the $\mathrm{VBC}_{8 / 9}$ and $\mathrm{FO}$ eigenstates of Hamiltonian (4). Along this line, the ground state is macroscopically degenerate, due to the dispersionless nature of the noninteracting lowest-energy band.

Below this saturation line, we find a staircase of $\mathrm{VBC}_{\rho}$ phases with fractional densities that dilutes into a CSF near half-filling, when diminishing the chemical potential. Similar to a devil staircase, smaller plateaux appear between the main ones at $\rho=5 / 9,2 / 3,7 / 9,8 / 9$, when increasing the cluster size from 9 to 27 sites. Moreover, these crystal phases prevail even in the absence of the density-density interaction, i.e., in the $\mathrm{XY}$ regime.

Note that the phase boundaries between main plateaux$\pi \mathrm{VBC}_{2 / 3}-\pi \mathrm{VBC}_{7 / 9}$ and $\pi \mathrm{VBC}_{7 / 9}-\mathrm{VBC}_{8 / 9}$ - computed with 9-HMFT almost coincide with the boundaries of the intermediate plateaux obtained with 27-HMFT. Also, notice the agreement between the lower boundary of the $\pi \mathrm{VBC}_{2 / 3}$ computed with 27-HMFT and the one obtained by 36-ED in all limits of the AFM regime.

When performing ED on different clusters we obtain various staircases, analogously to the FM regime. However, contrary to the FM regime, the main plateaux in the AFM regime do not decrease its width monotonically with increasing cluster size. This feature prevails in the XY regime, too. In the following, we give a detail analysis and comparison of the results obtained with both techniques in the AFM regime.

\section{HMFT approach}

When computing with 9-HMFT, the main plateaux with $\rho>5 / 9$ prevail up to the XY limit (see Fig. 8), and their transitions are all first order. The $\mathrm{VBC}_{5 / 9}$ plateau exhibits a lobe surrounded by a doubly degenerate CSF, having its tip at $t=1.1 \mathrm{~V}$. Upon increasing the cluster size to 27 sites, the main plateaux shrink leading to the appearance of other narrower plateaux characterized by densities commensurate with the cluster size. The $\mathrm{VBC}_{5 / 9}$ in this case extends to the $\mathrm{XY}$ limit, while the CSF region shrinks to near half-filling. Around halffilling and for small values of the hopping amplitude, $t<$ $0.4 \mathrm{~V}$, the CSF disappears giving rise to an additional VBC with density $\rho=14 / 27$, rendering the half-filling line for this region to be a first order transition line to a $\rho=13 / 27$ state.

These results are in partial disagreement with iPEPS results obtained in Ref. [8], where also a nine-site unit cell was used. There, U(1) symmetry broken phases were found in regions between the main plateaux, although not all regions were fully characterized. We cannot rigorously discard the possibility that the intermediate phase between the main plateaux may have a characteristic length scale larger than the clusters used in this work, and thus be characterized by either BEC condensation in k-points of the Brillouin zone different from the ones contained within the nine-site and 27-site clusters (see Fig. 4), or by a VBC with a periodicity not commensurate with our clusters, or other phase with topological order [42].

We next describe the main features of the principal $\mathrm{VBC}_{\rho}$ and CSF phases. Generically, the CB Gutzwiller wave function for a particular $\mathrm{VBC}_{\rho}$ depends exclusively on the hopping parameter $t$, and not on the chemical potential $\mu$. Moreover, it contains configurations with a definite number of hard-core bosons per cluster, $N_{\square}$. On the contrary, the CSF wave function, characterized by breaking of the global U(1) symmetry, i.e., onset of BEC, changes with both $\mu / V$ and $t / V$.

a. One-hole resonant state $\left(\mathrm{VBC}_{8 / 9}\right) . \mathrm{The}_{\mathrm{VBC}_{8 / 9}}$ phase is characterized by a fully stacked pattern of localized one-hole resonant states, which was shown to be one exact ground state of the XXZ Hamiltonian [9] along the line defined in Eq. (28). This state is exactly contained within the 9-HMFT Gutzwiller wave function and can be written as

$$
\left.\left|\Phi^{8 / 9}\right\rangle=\frac{1}{\sqrt{6}}\left(|\therefore . \therefore\rangle=-\mid \therefore . C_{3} \text { rots. }\right]\right) \text {. }
$$

Equivalently, $\left|\Phi^{8 / 9}\right\rangle$ be written as a state where a two-spin singlet resonates in the hexagon of the nine-site cluster.

$b$. Two-hole resonant state $\left(\pi \mathrm{VBC}_{7 / 9}\right)$. The $\mathrm{CB}$ Gutzwiller wave function $\pi \mathrm{VBC}_{7 / 9}$ is a localized two-hole resonant state that can be written in terms of a nine-site cluster as

$$
\begin{aligned}
\left|\Phi_{\pi}^{7 / 9}\right\rangle= & \beta_{1}\left(|\therefore\rangle+\left[C_{3} \text { rots. }\right]\right) \\
& \left.-\beta_{2}(|\therefore\rangle\rangle+\mid \therefore . \Omega\left(C_{3} \text { rots. }\right]\right) \\
& +\ldots,
\end{aligned}
$$

where $\beta_{1}$ and $\beta_{2}$ are positive real numbers, and the leading weights for the whole range up to the $X Y$ regime, i.e., 

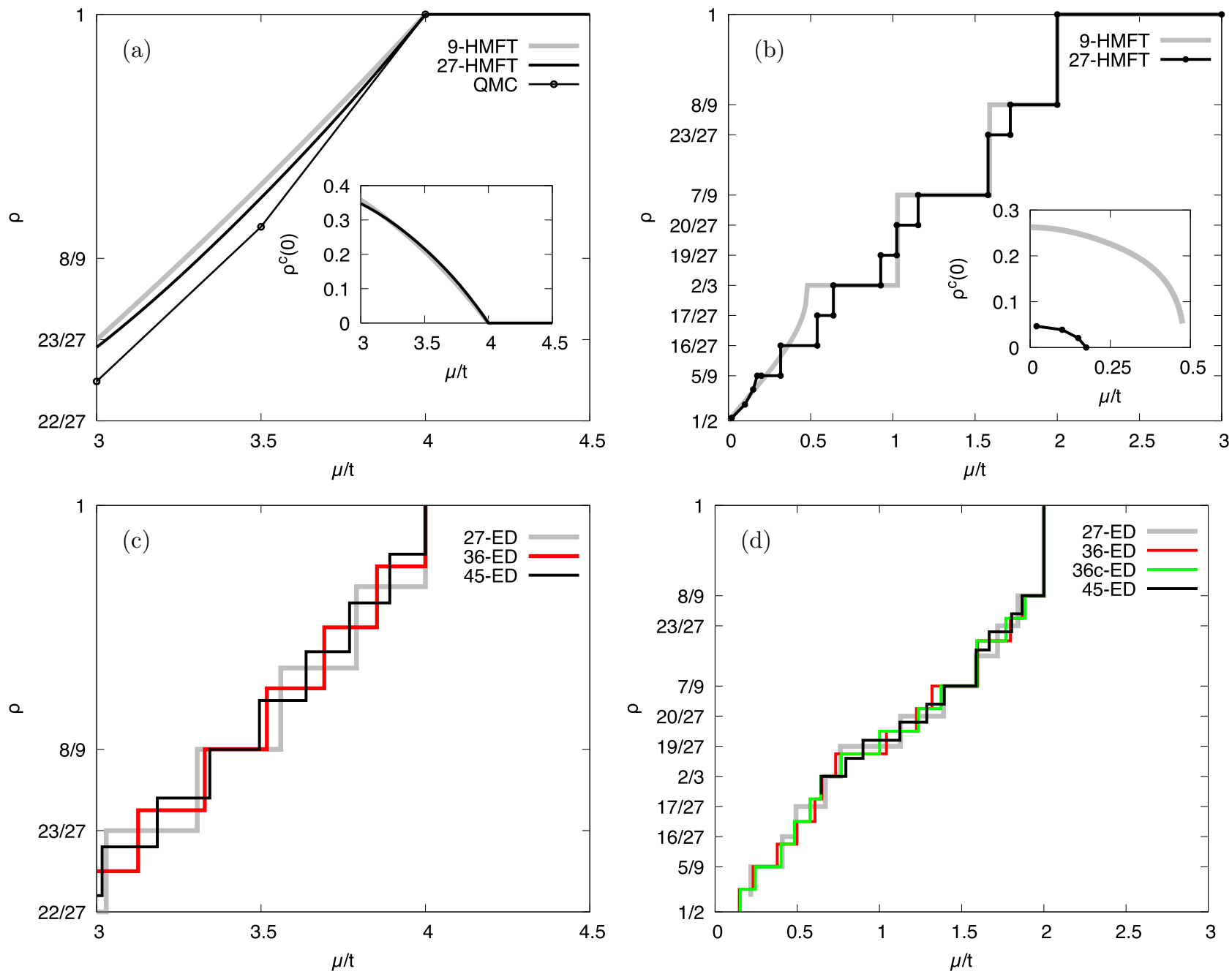

FIG. 8. Total density and condensate density (inset) in the XY limit $(V=0)$ for the (a) nonfrustrated regime $t<0$ and (b) frustrated regime $t>0$ computed with 9-HMFT and 27-HMFT. Total density for the (c) nonfrustrated and (d) frustrated regimes in the same XY limit obtained with ED. In the frustrated regime, the widths of the ED plateaux do not have a monotonic dependence with increasing cluster size, contrary to the nonfrustrated regime.

$\beta_{1}^{2}=0.16$ and $\beta_{1}^{2}=0.09$ for $t=0.1 \mathrm{~V}$, while $\beta_{1}^{2}=0.11$ and $\beta_{1}^{2}=0.08$ in the XY limit. This wave function, although not an exact eigenstate of the Hamiltonian (4), is a good approximation to the ground state. It reasonably describes the Heisenberg and XY limits, as can be seen by computing the expectation value of the $B_{i j}$ operator [defined in Eq. (21)] shown in Table I.

c. The $\pi \mathrm{VBC}_{2 / 3}$ state. Similarly to the $\mathrm{FM}$ regime, for small hopping amplitudes $(t<0.1 \mathrm{~V})$, the $\pi \mathrm{VBC}_{2 / 3}$ can be written within 9-HMFT as

$$
\left|\Phi_{\pi}^{2 / 3}\right\rangle=\gamma(|\therefore\rangle\rangle-\mid \therefore .
$$

with amplitude $\gamma^{2} \sim 0.49$, and the next leading term being two orders of magnitude smaller. This situation changes smoothly with increasing $t / V$, until the XY limit, where $\gamma^{2}=0.095$ and the next leading term is of the same order of magnitude. Near the Ising limit - and always at $\rho=2 / 3$ - the small hopping amplitude generates an effective three-body ring-exchange hopping which lifts the Ising macroscopic degeneracy and stabilizes VBC order [15]. Interestingly, the phase transition between the $\mathrm{VBC}_{2 / 3}$ and $\pi \mathrm{VBC}_{2 / 3}$ across the Ising line is not first order, but continuous, as can be seen by inspecting the energy and its second-order derivative in Fig. 9. This is probably due to the large number of quasidegenerate low-lying $\pi \mathrm{VBC}_{2 / 3}$ states present in the frustrated regime.

For $t>0.1 \mathrm{~V}$, many other configurations with $N_{\square}=6$ start to have relevant weights, and thus the wave function cannot be approximated by a three-hole resonant state (31). This fact results in a complex VBC pattern, as can be seen when inspecting the expectation value of the $B_{i j}$ operator computed with 27-HMFT (Table I). Interestingly, in the XY limit, this phase was argued to be a fractional quantum Hall state characterized by nontrivial topological order, based on a Chern-Simons analysis [42].

$d$. The $\pi \mathrm{VBC}_{5 / 9}$ state. Leading contributions to this state cannot be interpreted in terms of resonant holes over a background of fixed particles in any regime of the phase diagram. This is also evident in the related pattern of expectation values of the $B_{i j}$ operator in Table I. Interestingly, this phase was argued to be a translational invariant gapped phase with $\mathbb{Z}_{3}$ 
TABLE I. Expectation value of the $B_{i j}$ operator (21) for the Heisenberg and $\mathrm{XY}$ limits, in the frustrated regime, within main $\mathrm{VBC}_{\rho}$ plateaux. Thickness of the bonds is proportional to the absolute value of $\left\langle B_{i j}\right\rangle$. For the 27-HMFT, the maximum absolute value is 0.4 and blue (red) color refers to its negative (positive) sign. Notice that only the $\pi \mathrm{VBC}_{5 / 9}$ exhibits some slightly positive values for three of the bonds in the cluster.
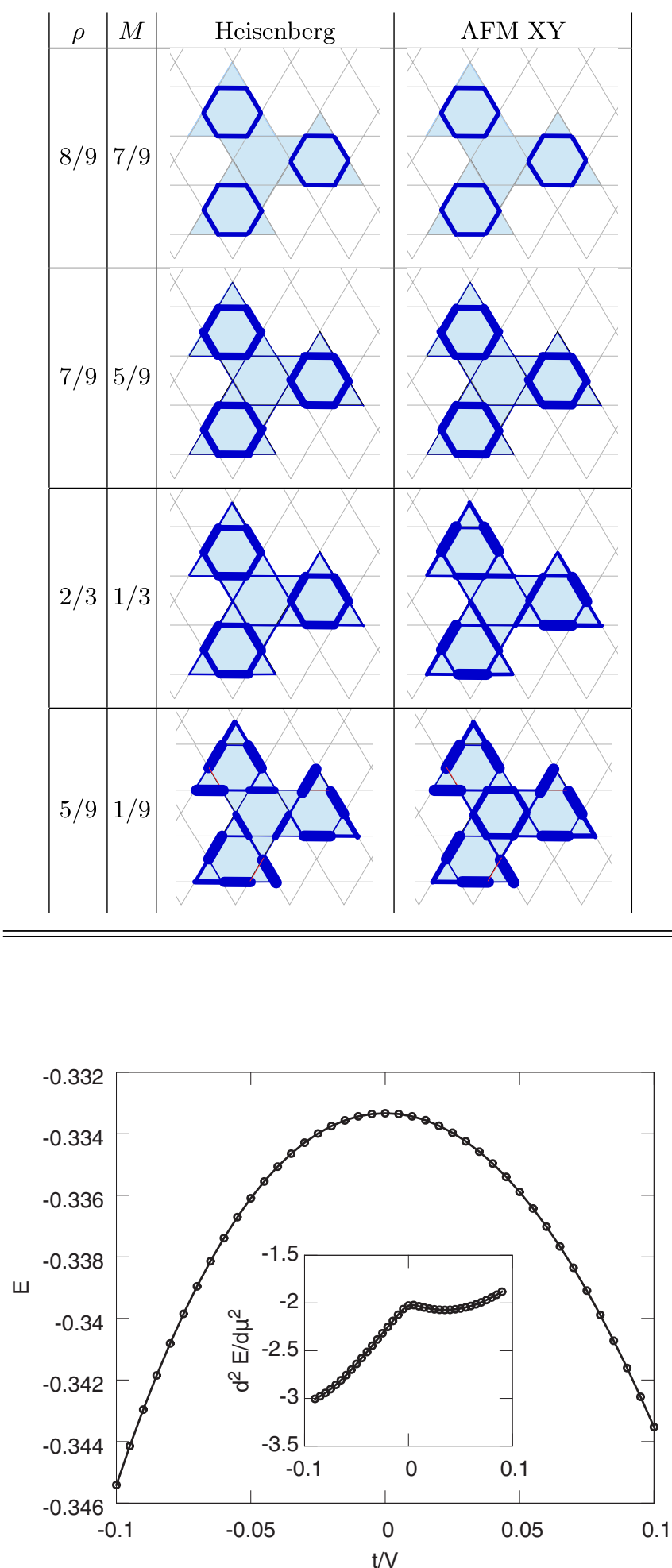

FIG. 9. Energy (in units of $V$ ) and second-order derivative (inset) across the $\pi \mathrm{VBC}_{2 / 3}$ to $\mathrm{VBC}_{2 / 3}$ transition for $\mu=3 V$. topological order, based on a DMRG study [7], and a VBC phase with 18-fold degeneracy, based on an iPEPS study [8] Further analysis of the phase based on ED is presented below.

e. Chiral superfluid phase. The doubly degenerate CSF is characterized by breakdown of the U(1) symmetry, signaled by a finite $\mathrm{BEC}$ at $\Gamma$, and nonvanishing bond currents where triangles and hexagons host vortices with opposite charge. Additionally, it possesses null total transverse magnetization per cluster, i.e., $\left\langle\Psi\left|\sum_{j \in \square} \mathbf{S}_{j}^{x}\right| \Psi\right\rangle=0=\left\langle\Psi\left|\sum_{j \in \square} \mathbf{S}_{j}^{y}\right| \Psi\right\rangle$, a common feature with previous iPEPS studies in Ref. [8]. Both the chirality (shown in Fig. 10) and condensate density are nevertheless suppressed upon increasing the cluster size from 9-HMFT to 27-HMFT, and affected by finite-size effects, as three of the triangles in the 27-site cluster change the sense of chirality.

To assess the stability of the chiral order around half-filling, we have performed 18-HMFT computations ${ }^{2}$ along the halffilling line, finding a SF in the AFM XY regime that transitions to a half-filled $\mathrm{VBC}_{1 / 2}$ at $t \sim V$, both states without chiral order. The relation between the presence (absence) of chirality and the odd (even) number of sites of the cluster is consistent with previous ED calculations of the KHAF, demonstrating the existence of low-lying $S=1$ states characterized by nontrivial (null) Chern numbers-related to breaking of time reversalwhen using odd (even) clusters [19]. Therefore we conjecture that the nonvanishing chirality of the CSF found with 9- and 27-HMFT reflects the admixture of these nontrivial excited states when using clusters with an odd number of sites.

Moreover, from an energetics viewpoint, the CSF is competing with several VBC phases (the ones with odd fillings are doubly-degenerate due to particle-hole symmetry), indicating that it may eventually be replaced by a degenerate manifold of phases in the thermodynamic limit. In particular, at the KHAF point, the energies per site-i.e., the expectation value of (4) for $t / V=1 / 2$ and $\mu / V=2-$ obtained with 9-, 18-, and 27-HMFT and various cutoffs (27-HMFT) are

$$
\begin{aligned}
E_{9}=-0.4006, & \operatorname{CSF}, \\
E_{27}=-0.4112, & \pi \mathrm{VBC}_{5 / 9} \quad \text { and } \pi \mathrm{VBC}_{4 / 9}, \\
E_{18}=-0.4135, & \operatorname{VBC}_{1 / 2}, \\
E_{27}=-0.4148, & \operatorname{CSF}\left(13 \leqslant N_{\square} \leqslant 14\right), \\
E_{27}=-0.4169, & \operatorname{VBC}_{13 / 27} \quad \text { and } \operatorname{VBC}_{14 / 27}, \\
E_{27}=-0.4175, & \operatorname{CSF}\left(12 \leqslant N_{\square} \leqslant 15\right),
\end{aligned}
$$

in units of $V$. These values are consistent with those obtained by the computationally demanding tensor network techniques, such as the projected entangled simplex states (PESS), where it was found an energy $E_{\mathrm{PESS}}=-0.4364$ (1) for a nine-site cell with bond dimension $D=13$, and a translationally invariant phase with no broken symmetry [52].

\section{Exact diagonalization approach}

Using ground-state energies for fixed densities and different cluster sizes and shapes, i.e. 27-, 36-, 36c-, and 45-ED (see

\footnotetext{
${ }^{2}$ The 18 -site cluster used is comprised of two vertically connected nine-site clusters.
} 


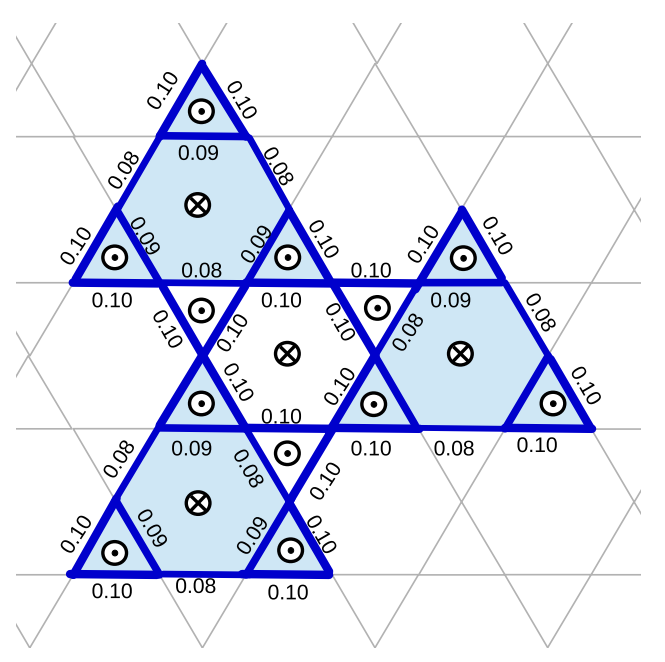

(a)

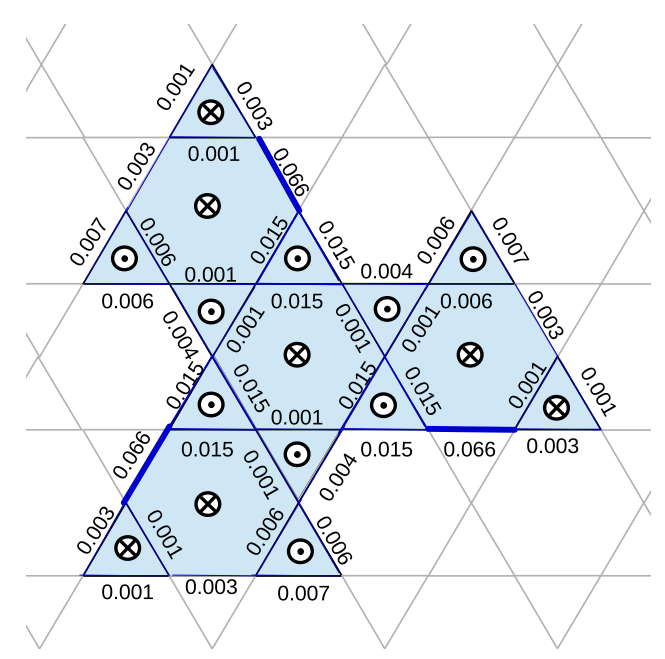

(b)

FIG. 10. Absolute value of the expectation of the $\mathcal{J}_{i j}$ operator defined in Eq. (18) in the CSF phase as computed with (a) 9-HMFT, and (b) 27-HMFT, in the AFM XY limit at $\mu / t=0.02, V=0$. Thickness of the blue lines is proportional to $\left|\left\langle\mathcal{J}_{i j}\right\rangle\right|$, and its maximum value is $\left|\left\langle\mathcal{J}_{i j}\right\rangle\right|=0.1$. The chirality of the loop currents is represented by a circled point (vortex) or a crossed circle (antivortex).

Fig. 5), we have computed the density as a function of the chemical potential using a Legendre transform for different values of the hopping amplitude. In particular, the widths of the main plateaux for $t / V=0.1,0.5,1.0$, and 1.5 computed with a 36-ED cluster in the AFM regime are included in the general phase diagram (Fig. 6). Results on the density staircases as a function of the chemical potential are plotted in Fig. 8(c) and 8(d) for both the FM and AFM XY regimes, respectively. Due to the low number of cluster sizes containing the exact $\mathrm{VBC}_{8 / 9}$ available with current computational capabilities, performing an appropriate finite-size scaling analysis is difficult, thus we restrict ourselves to the analysis of the main plateaux $\rho=$ 5/9, 2/3, 7/9, 8/9.

As a first attempt to characterizing the phase diagram, and specially to assess whether an adiabatic continuity from the noninteracting $V=0(\mathrm{XY})$ regime to the classical limit $t=0$ (Ising) exists, we computed bond-bond correlators (22), shown in Table II, as well as the excitation energy spectrum as a function of the density in both regimes, shown in Fig. 11.

a. One-hole resonant state $\left(\mathrm{VBC}_{8 / 9}\right)$. At $\rho=8 / 9$, both in the Ising and $\mathrm{XY}$ regimes, there exists an exact threefold degeneracy due to the existence of an exact localized magnon eigenstate [9] for any regime of the interaction $V$. Due to the particular shape of the 36-ED cluster, small loops go around the cluster, and we find a greater degeneracy. However, on larger clusters (e.g., 63-ED), the exact threefold degeneracy with expected quantum numbers is recovered [6]. From general considerations, the $\mathrm{VBC}_{8 / 9}$ should possess gapped excitations and hence correspond to an extended region in the phase diagram.

b. Two-hole resonant state $\left(\pi \mathrm{VBC}_{7 / 9}\right)$. At $\rho=7 / 9$, the spectra both at the Ising and $\mathrm{XY}$ regimes exhibit a twofold degenerate state at momentum $K \mathrm{~A}_{1}$ very close to the ground state $\left(\Gamma A_{1}\right)$, and then a rather large gap above them. Such data suggests that this first excited state may probably collapse
TABLE II. Kinetic bond-bond correlations (22) computed by ED on a $N=36$ cluster at $t=0.1 V$ (near Ising), and the AFM XY limit. Positive (negative) values are shown with blue (red) lines and their width is proportional to the data, the reference bond being shown in black.

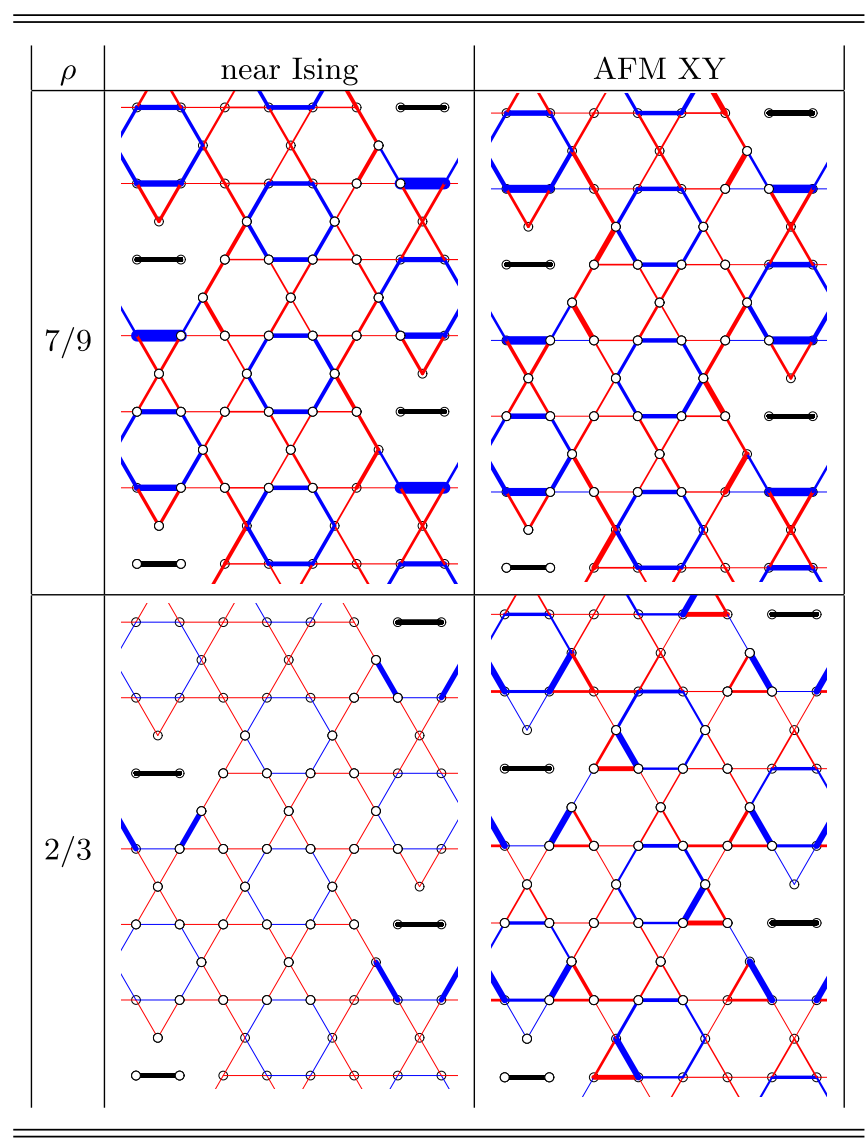


(a)

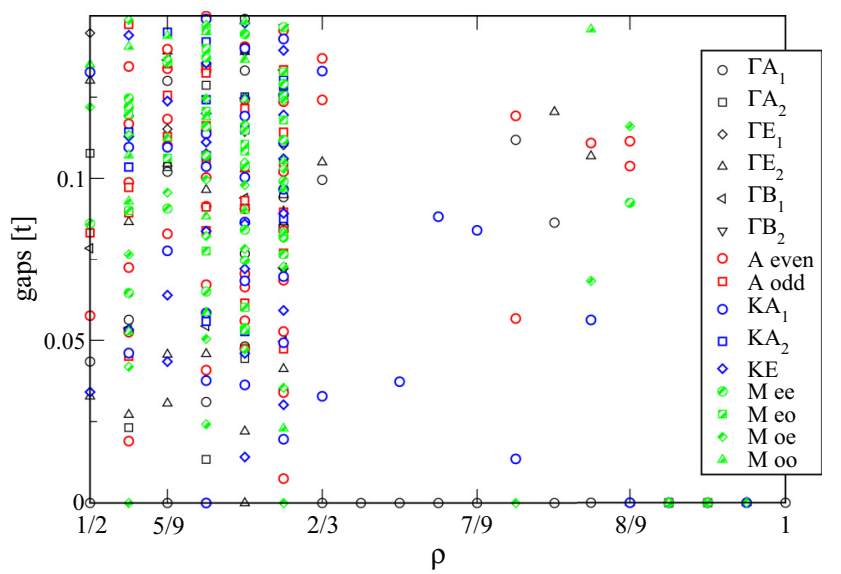

(b)

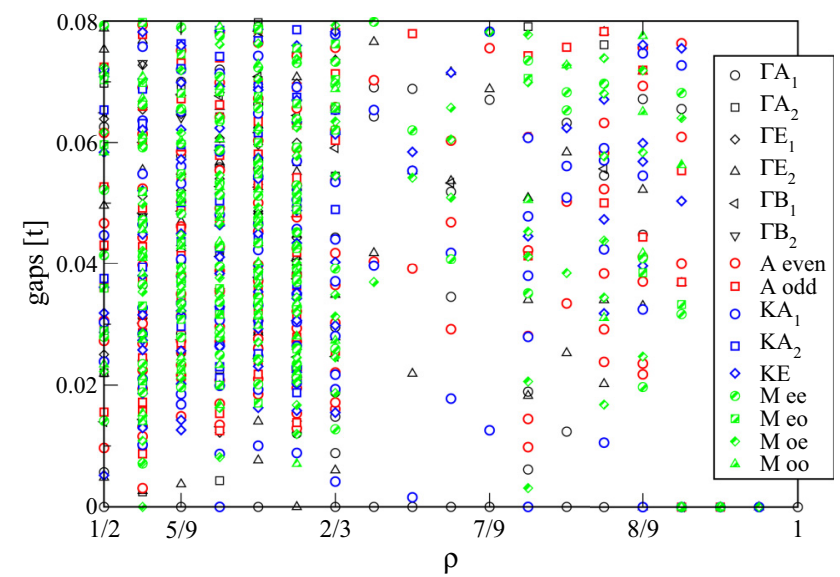

FIG. 11. Tower of states representing excitation energies (in units of $2 t$ ) as a function of density $\rho$, obtained by ED on a $N=36$ cluster. States are labelled with respect to their quantum numbers associated to translation and point group symmetry (see Ref. [6] for more details): (a) $t=0.1 V$ (near Ising regime) and (b) $V=0$ (AFM XY).

to the ground state in the thermodynamic limit, stabilizing a threefold degenerate VBC equivalent to the one found with HMFT at this density (see Table I). Further computations of the kinetic energy correlator (22), shown in Table II, support this result.

Moreover, the $\pi \mathrm{VBC}_{7 / 9}$ found in this limit is equivalent to the one found at the Heisenberg limit in previous studies [6,7], suggesting that it is stable in the whole AFM regime. On the contrary, we find no evidence of any additional degeneracy that would signal either an additional symmetry breaking, as proposed in Ref. [8], or the stabilization of a translational invariant gapped phase with topological order, as proposed in Ref. [42].

c. The $\pi \mathrm{VBC}_{2 / 3}$ state. At $\rho=2 / 3$ and in the Ising limit, there are clear signatures of the classical degeneracy expected for the $\pi \mathrm{VBC}_{2 / 3}$ state. The first excited state over the ground state $\left(\right.$ at $\left.\Gamma \mathrm{A}_{1}\right)$ is at momentum $K \mathrm{~A}_{1}$ (twofold degenerate) while the next excited state is far in energy. Again, this suggests that this first excited state may collapse onto the ground state in the thermodynamic limit, giving a threefold broken translational symmetry state, in agreement with the results obtained with HMFT.

However, in the XY regime, there is no clear separation of states in the low-energy spectrum. This may signal the stabilization in the thermodynamic limit of either a VBC state with larger unit cell and larger degeneracy (in case the first excited state $K \mathrm{~A}_{1}$ collapsed onto the ground state), or an eventual translational invariant gapped phase with manifold degeneracy (in case the $\Gamma \mathrm{E}_{2}$ would collapse but not the $K \mathrm{~A}_{1}$ ), or even the breakdown of global U(1) (gapless phase) by BEC at some $\mathbf{k}$ point not commensurate with the clusters utilized in HMFT. Note, nevertheless, that the width of the plateau at this density increases when increasing the cluster size from 36 to 45 [see Fig. 8(d)].

To further discuss the existence of an adiabatic continuity connecting the Ising, Heisenberg and XY regimes of the main plateaux in the AFM regime of the phase diagram, we show in Fig. 12 the fidelity susceptibility $\chi_{F}$ per site defined in Eq. (24).
The size independence for $\rho=7 / 9$ is in agreement with the stability of the $\pi \mathrm{VBC}_{7 / 9}$ over all regimes of interaction as found with HMFT. However, for $\rho=2 / 3$, the increase of $\chi_{F}$ with system size may signal a quantum phase transition in the vicinity of the AFM XY limit where, coincidently, the VBC pattern obtained with HMFT is no more of the localized resonant-magnon type. Larger clusters are required to reach a definite conclusion.

Similarly, in Table II, we show kinetic bond-bond correlations (22) for $\rho=7 / 9$ and $\rho=2 / 3$ in both the AFM XY and Ising limits. Although the numerical data shows different amplitudes and short-range features, the overall pattern is compatible with the $\pi \mathrm{VBC}_{7 / 9}$ and $\pi \mathrm{VBC}_{2 / 3}$, respectively, known to be the ground states in the Ising [15] and Heisenberg $[6,7]$ limits.

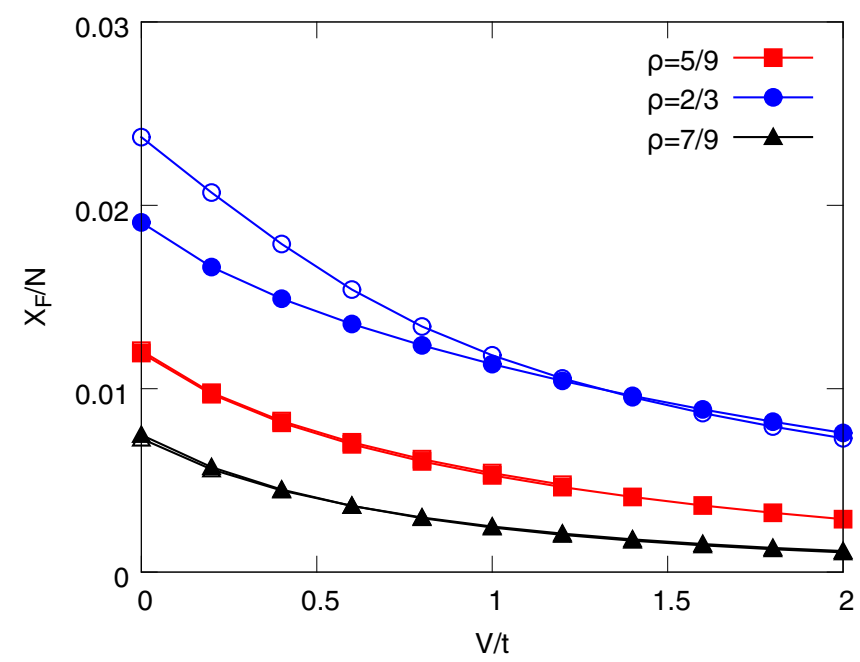

FIG. 12. Fidelity susceptibility per site $\chi_{F} / N(24)$ as a function of $V / t$ from the XY regime to the $\mathrm{SU}(2)$ point, computed by 27-ED (filled) and 36-ED (empty) for various densities. For $\rho=5 / 9$ and 7/9 filled and empty symbols are superimposed. 
In summary, it was claimed in previous ED studies [15] that the ground state at $\rho=2 / 3$ is qualitatively similar for any $V>0$, i.e., of the same VBC nature as found in the Ising limit. From our ED data, we cannot conclusively establish the exact nature of the $\rho=2 / 3$ plateau in the XY limit, leaving the question of whether it is a more complex-pattern VBC (see Table I) or a gapped topological state [42] for future studies.

$d$. The $\pi \mathrm{VBC}_{5 / 9}$ state. From the fidelity measurement (Fig. 12), we do not find any sign of a quantum phase transition taking place within the $\pi \mathrm{VBC}_{5 / 9}$ plateau when varying the density-density interaction strength $V$. From our results, we conclude that the ground state in the XY limit should be similar to the one encountered at the Ising and Heisenberg limits.

Regarding the ED low-energy spectrum in the XY limit at this density (see Fig. 11), we identify a twofold energy state at the $\Gamma$ point $\left(\Gamma E_{2}\right)$ very close to the ground state, and a tower of close states well above. This may result in an eventual collapse to the ground state in the thermodynamic limit, stabilizing a translational invariant threefold degenerate ground state, which would be compatible with either a topological state [7] or a peculiar VBC that would only break point-group symmetry but not translations.

Interestingly, in the Heisenberg limit, this $\pi \mathrm{VBC}_{5 / 9}$ phase has been claimed to be a threefold degenerate topological state, based on DMRG studies [7], and to be an 18-fold degenerate VBC, based on iPEPS computations with a nine-site cell [8]. Our HMFT results also predicts a VBC with the same degree of degeneracy as the one of Ref. [8], but with a different bond pattern (see Table I).

\section{SUMMARY AND CONCLUSIONS}

We have determined the quantum phase diagram of a system of repulsively interacting hard-core bosons on the kagome lattice by means of the hierarchical mean field theory (HMFT) and exact diagonalization (ED) techniques. The system is isomorphic to the spin $S=1 / 2 \mathrm{XXZ}$ model on the kagome lattice in presence of an external magnetic field, a paradigmatic example of frustrated magnetism, and relevant to unveil magnetic properties of Mott insulating materials. We have studied the nonfrustrated (ferromagnetic), and frustrated (antiferromagnetic) regimes, ranging from the purely repulsive (Ising) to the noninteracting (XY) limits.

In the nonfrustrated regime, we found two valence bond crystal $\left(\mathrm{VBC}_{\rho}\right)$ lobes at densities $\rho=1 / 3$ and $\rho=2 / 3$ that melt into a standard superfluid characterized by the onset of Bose-Einstein condensation at momentum $\mathbf{k}=0$, in semiquantitative agreement with previous quantum Monte Carlo (QMC) results.

In the frustrated regime, where QMC computations are impeded by the sign-problem, and away from half-filling, we have found a series of wide plateaux with $\mathrm{VBC}_{\rho}$ order at densities $\rho$ commensurate with $1 / 9$, in agreement with previous numerical studies carried out on the Heisenberg line (DMRG [7], ED [6], iPEPS [8]). These main plateaux are separated by narrower plateaux at fillings commensurate with $1 / 27$, when using clusters with 27 sites. The physical mechanism responsible for the density (magnetization) plateaux is similar to the one found for the, in principle unrelated,
Shastry-Sutherland model [53,54]. Moreover, near half-filling this devil staircase of crystal phases melts into a superfluid phase characterized by a Bose-Einstein condensate (BEC). Interestingly, we discovered a peculiar "odd-even effect": the superfluid is chiral (nonchiral) whenever the size of the simulated cluster is odd (even). Note, however, that several $\mathrm{VBC}_{\rho}$ phases compete in energy near this half-filling region. The devil staircase physics and its melting mechanism prevail essentially up to the antiferromagnetic XY limit. This shows that correlations are adiabatically continued within a quantum phase as the hopping (or transverse exchange interaction in the spin language) changes from the Ising to the $\mathrm{XY}$ limits.

In particular, the plateaux at $\rho=8 / 9$ and $\rho=7 / 9\left(\mathrm{VBC}_{8 / 9}\right.$ and $\pi \mathrm{VBC}_{7 / 9}$, respectively) are characterized by a fully stacked pattern of localized resonant magnons in all regimes. The plateau at $\rho=2 / 3\left(\pi \mathrm{VBC}_{2 / 3}\right)$ is also characterized by three localized resonant magnons, except for the antiferromagnetic XY limit, where we found a more complex VBC pattern. Based on the analysis of the low-lying ED energy spectrum of 36-site clusters, and the fidelity susceptibility for this density, we cannot exclude that the XY limit may also be described by a gapped translational invariant phase in the thermodynamic limit, or even a gapless BEC. Similarly, the plateau at $\rho=5 / 9$ is found to be a complex $\mathrm{VBC}_{\rho}\left(\pi \mathrm{VBC}_{5 / 9}\right)$ that changes smoothly from the Ising and Heisenberg regimes to the antiferromagnetic XY limit. Further analysis on the low-lying ED energy spectrum of 36-site clusters reveals the possibility of stabilization, in the thermodynamic limit, of a threefold degenerate gapped translational invariant state, which would not be inconsistent with a proposed topological order [7].

We note that, based on the 36-ED tower of states (Fig. 11) and the HMFT analysis, one can clearly distinguish two regimes with respect to the density of low-lying excited states. For $\rho<2 / 3$, the density of low-lying excited states is much higher than for $\rho>2 / 3$. This, together with the fact that the lower- $\mu \pi \mathrm{VBC}_{2 / 3}$ phase boundary computed with $27-\mathrm{HMFT}$ and with 36-ED coincide (see Fig. 6), suggest that, in the thermodynamic limit, for densities $\rho \leqslant 2 / 3$ the staircase may be comprised of an infinite series of gapped phases. It is clear that quantum fluctuations become more relevant as we move towards the half-filled and noninteracting limits of the quantum phase diagram.

Note added. After submission of the current manuscript, we noted the work of Ref. [55] that studied the same model and finds an $M=1 / 3$ plateau in the entire frustrated regime.

\section{ACKNOWLEDGMENTS}

D.H. gratefully acknowledge the computing time granted on the supercomputer JURECA at Jülich Supercomputing Centre (JSC). D.H. and J.D. acknowledge support from the Spanish Ministry of Economy and Competitiveness through Grants FIS2012-34479 and FIS2015-63770-P (MINECO/FEDER). S.C. would like to acknowledge HPC resources from GENCI (Grants x2015050225 and $\mathrm{x} 2016050225$ ) and CALMIP (Grants 2015-P0677 and 2016-P0677). The QMC SSE simulations were performed using the code from the ALPS libraries [35]. 
[1] C. Lacroix, P. Mendels, and F. Mila, Introduction to Frustrated Magnetism: Materials, Experiments, Theory (Springer, Verlag, 2011).

[2] M. Takigawa, M. Horvatić, T. Waki, S. Krämer, C. Berthier, F. Lévy-Bertrand, I. Sheikin, H. Kageyama, Y. Ueda, and F. Mila, Phys. Rev. Lett. 110, 067210 (2013).

[3] H. Nishimori and G. Ortiz, Elements of Phase Transitions and Critical Phenomena (Oxford University Press, Oxford, 2011).

[4] M. Roger, J. H. Hetherington, and J. M. Delrieu, Rev. Mod. Phys. 55, 1 (1983).

[5] P. Bak, Rep. Prog. Phys. 45, 587 (1982).

[6] S. Capponi, O. Derzhko, A. Honecker, A. M. Läuchli, and J. Richter, Phys. Rev. B 88, 144416 (2013).

[7] S. Nishimoto, N. Shibata, and C. Hotta, Nat. Commun. 4, 3287 (2013).

[8] T. Picot, M. Ziegler, R. Orús, and D. Poilblanc, Phys. Rev. B 93, 060407 (2016).

[9] J. Schulenburg, A. Honecker, J. Schnack, J. Richter, and H.-J. Schmidt, Phys. Rev. Lett. 88, 167207 (2002).

[10] M. P. Shores, E. A. Nytko, B. M. Bartlett, and D. G. Nocera, J. Am. Chem. Soc. 127, 13462 (2005).

[11] J. S. Helton, K. Matan, M. P. Shores, E. A. Nytko, B. M. Bartlett, Y. Yoshida, Y. Takano, A. Suslov, Y. Qiu, J.-H. Chung, D. G. Nocera, and Y. S. Lee, Phys. Rev. Lett. 98, 107204 (2007).

[12] P. Mendels, F. Bert, M. A. de Vries, A. Olariu, A. Harrison, F. Duc, J. C. Trombe, J. S. Lord, A. Amato, and C. Baines, Phys. Rev. Lett. 98, 077204 (2007).

[13] M. Fu, T. Imai, T.-H. Han, and Y. S. Lee, Science 350, 655 (2015).

[14] G.-B. Jo, J. Guzman, C. K. Thomas, P. Hosur, A. Vishwanath, and D. M. Stamper-Kurn, Phys. Rev. Lett. 108, 045305 (2012).

[15] D. C. Cabra, M. D. Grynberg, P. C. W. Holdsworth, A. Honecker, P. Pujol, J. Richter, D. Schmalfuß, and J. Schulenburg, Phys. Rev. B 71, 144420 (2005).

[16] S. V. Isakov, S. Wessel, R. G. Melko, K. Sengupta, and Y. B. Kim, Phys. Rev. Lett. 97, 147202 (2006).

[17] M. Mambrini and F. Mila, Eur. Phys. J. B 17, 651 (2000).

[18] D. Poilblanc, M. Mambrini, and D. Schwandt, Phys. Rev. B 81, 180402 (2010).

[19] C. Waldtmann, H.-U. Everts, B. Bernu, C. Lhuillier, P. Sindzingre, P. Lecheminant, and L. Pierre, Eur. Phys. J. B 2, 501 (1998).

[20] R. R. P. Singh and D. A. Huse, Phys. Rev. B 76, 180407 (2007).

[21] S. Yan, D. A. Huse, and S. R. White, Science 332, 1173 (2011).

[22] S. Depenbrock, I. P. McCulloch, and U. Schollwöck, Phys. Rev. Lett. 109, 067201 (2012).

[23] H.-C. Jiang, Z. Wang, and L. Balents, Nat. Phys. 8, 902 (2012).

[24] L. Messio, B. Bernu, and C. Lhuillier, Phys. Rev. Lett. 108, 207204 (2012).

[25] S. Capponi, V. R. Chandra, A. Auerbach, and M. Weinstein, Phys. Rev. B 87, 161118 (2013).

[26] Y. Iqbal, F. Becca, S. Sorella, and D. Poilblanc, Phys. Rev. B 87, 060405 (2013).
[27] T. Matsubara and H. Matsuda, Prog. Theor. Phys. (Kyoto) 16, 569 (1956).

[28] G. Ortiz and C. D. Batista, Phys. Rev. B 67, 134301 (2003).

[29] C. D. Batista and G. Ortiz, Adv. Phys. 53, 1 (2004).

[30] L. Isaev, G. Ortiz, and J. Dukelsky, Phys. Rev. B 79, 024409 (2009).

[31] L. Isaev and G. Ortiz, Phys. Rev. B 86, 100402(R) (2012).

[32] D. Huerga, J. Dukelsky, N. Laflorencie, and G. Ortiz, Phys. Rev. B 89, 094401 (2014).

[33] A. W. Sandvik and J. Kurkijärvi, Phys. Rev. B 43, 5950 (1991).

[34] O. F. Syljuåsen and A. W. Sandvik, Phys. Rev. E 66, 046701 (2002).

[35] B. Bauer, L. D. Carr, H. G. Evertz, A. Feiguin, J. Freire, S. Fuchs, L. Gamper, J. Gukelberger, E. Gull, S. Guertler, A. Hehn, R. Igarashi, S. V. Isakov, D. Koop, P. N. Ma, P. Mates, H. Matsuo, O. Parcollet, G. Pawłowski, J. D. Picon, L. Pollet, E. Santos, V. W. Scarola, U. Schollwöck, C. Silva, B. Surer, S. Todo, S. Trebst, M. Troyer, M. L. Wall, P. Werner, and S. Wessel, J. Stat. Mech. (2011) P05001.

[36] V. G. Rousseau, Phys. Rev. B 90, 134503 (2014).

[37] S.-S. Gong, W. Zhu, and D. N. Sheng, Sci. Rep. 4, 6317 (2014).

[38] B. Bauer, L. Cincio, B. P. Keller, M. Dolfi, G. Vidal, S. Trebst, and A. W. W. Ludwig, Nat. Commun. 5, 5137 (2014).

[39] Y.-C.He, D. N. Sheng, and Y. Chen, Phys. Rev. Lett. 112, 137202 (2014).

[40] A. M. Läuchli and R. Moessner, arXiv:1504.04380 [condmat.quant-gas].

[41] Y.-C. He and Y. Chen, Phys. Rev. Lett. 114, 037201 (2015).

[42] K. Kumar, K. Sun, and E. Fradkin, Phys. Rev. B 90, 174409 (2014).

[43] S. Greschner, D. Huerga, G. Sun, D. Poletti, and L. Santos, Phys. Rev. B 92, 115120 (2015).

[44] D. Huerga, J. Dukelsky, and G. E. Scuseria, Phys. Rev. Lett. 111, 045701 (2013).

[45] A. J. Leggett, Rev. Mod. Phys. 73, 307 (2001).

[46] K. A. Al-Hassanieh, C. D. Batista, G. Ortiz, and L. N. Bulaevskii, Phys. Rev. Lett. 103, 216402 (2009).

[47] S.-J. Gu, Int. J. Mod. Phys. B 24, 4371 (2010).

[48] A. F. Albuquerque, F. Alet, C. Sire, and S. Capponi, Phys. Rev. B 81, 064418 (2010).

[49] L. Jiang and J. Ye, J. Phys.: Condens. Matter 18, 6907 (2006).

[50] K. Sengupta, S. V. Isakov, and Y. B. Kim, Phys. Rev. B 73, 245103 (2006).

[51] K. Damle and T. Senthil, Phys. Rev. Lett. 97, 067202 (2006).

[52] Z. Y. Xie, J. Chen, J. F. Yu, X. Kong, B. Normand, and T. Xiang, Phys. Rev. X 4, 011025 (2014).

[53] B. S. Shastry and B. Sutherland, Physica B+C 108, 1069 (1981).

[54] L. Isaev, G. Ortiz, and J. Dukelsky, Phys. Rev. Lett. 103, 177201 (2009).

[55] A. Kshetrimayum, T. Picot, R. Orus, and D. Poilblanc, arXiv:1608.00437 [cond-mat.str-el]. 